This item was submitted to Loughborough's Research Repository by the author.

Items in Figshare are protected by copyright, with all rights reserved, unless otherwise indicated.

\title{
Numerical modelling of damage initiation in low-density thermally bonded nonwovens
}

PLEASE CITE THE PUBLISHED VERSION

http://dx.doi.org/10.1016/j.commatsci.2012.05.038

PUBLISHER

(C) Elsevier B.V.

VERSION

AM (Accepted Manuscript)

\section{PUBLISHER STATEMENT}

This work is made available according to the conditions of the Creative Commons Attribution-NonCommercialNoDerivatives 4.0 International (CC BY-NC-ND 4.0) licence. Full details of this licence are available at: https://creativecommons.org/licenses/by-nc-nd/4.0/

\section{LICENCE}

CC BY-NC-ND 4.0

\section{REPOSITORY RECORD}

Farukh, Farukh, Emrah Demirci, Baris Sabuncuoglu, Memis Acar, Behnam Pourdeyhimi, and Vadim V. Silberschmidt. 2019. "Numerical Modelling of Damage Initiation in Low-density Thermally Bonded Nonwovens”. figshare. https://hdl.handle.net/2134/16997. 


\section{Numerical Modelling of Damage Initiation in Low-Density Thermally Bonded Nonwovens}

*Farukh Farukh ${ }^{(1)}$, Emrah Demirci ${ }^{(1)}$, Baris Sabuncuoglu ${ }^{(1)}$, Memiş Acar ${ }^{(1)}$, Behnam Pourdeyhimi (2), Vadim V. Silberschmidt ${ }^{(1)}$

1 - Wolfson School of Mechanical and Manufacturing Engineering, Loughborough University, UK 2 - Nonwovens Cooperative Research Center, North Carolina State University, Raleigh, NC, USA *Corresponding author's e-mail: F.Farukh@lboro.ac.uk Tell: 004475863680541; fax: 00441509227585

Add: Wolfson School of Mechanical and Manufacturing Engineering, Loughborough University, UK

Keywords: nonwoven; anisotropy; finite element; damage

Abstract: Due to random orientation of fibres and presence of voids in their microstructure, lowdensity thermally bonded polymer-based nonwovens demonstrate complex processes of deformation and damage initiation and evolution. This paper aims to introduce a micro-scale discontinuous finite element model to simulate an onset of damage in low-density nonwovens. In the model, structural randomness of a nonwoven fabric was introduced in terms of orientation distribution function (ODF) obtained by an algorithm based on the Hough Transform. Fibres were represented in the model with truss elements with orientations defined according to the computed ODF. Another structural element of nonwovens -bond points- were modelled with shell elements having isotropic mechanical properties. The numerical scheme employed direct modelling of fibres at micro level, naturally introducing the presence of voids into the model and thus making it suitable for simulations of low-density nonwovens. The obtained results of FE simulations were compared with our data of tensile tests performed in principal directions until the onset of damage in the specimens.

\section{Introduction}

Several advantages are attributed to nonwovens compared to conventional textiles. The most important one is that the control over the properties of nonwovens during their manufacturing is easier than for woven fabrics. Besides, they are cheaper and can be manufactured with desired characteristics at higher speeds [1]. Therefore, the application spectrum of nonwovens has increased to such an extent that their usage now ranges from daily-life products, such as personal hygiene to high-tech applications, for instance in aerospace industry. All of these applications require integrity 
of nonwoven structure during their service life. Hence, it is important to understand the deformation and damage behaviours of nonwovens so that they could be produced to perform their intended functions without being damaged when exposed to service loading conditions.

Nonwovens are made of randomly-oriented fibres consolidated in a pattern by mechanical, chemical or thermal methods and thus demonstrate randomness of their microstructure. As a result of this randomness, nonwoven materials have a unique deformation and damage behaviours, different from those of woven ones [2].

The type of nonwovens studied in this work is a low-density thermally bonded fabric made of polypropylene fibres. Numerical modelling of deformation behaviour of such nonwovens has been a challenging task due to their randomness, discontinuity and presence voids in their microstructure. Only a few studies related to numerical modelling of low-density nonwovens are available in literature. Models with periodic symmetric microstructure were presented in [3, 4]; these models do not represent the realistic random microstructure of nonwovens. Some recent works introduced the distribution of fibre orientation into the models [5-10]. However, they can simulate only the initial deformation stage of nonwoven materials; none of these numerical models can predict the damage behaviour of nonwovens. From a mechanical viewpoint, damage is inevitable when material is subjected to an excessive mechanical loading. Thus, it is vital to understand the micromechanisms of damage, which control the behaviour of these materials after a certain level of loading and to develop a finite element model to simulate the damage behaviour.

In finite element modelling of low-density nonwovens, development of material microstructure with actual orientation distribution of fibres is the most challenging task. This task was solved with the help of a parametric modelling technique based on the developed user subroutine. Properties of two regions of the studied fabric - fibrous web and bond points - necessary for FE modelling of the material, were computed using the behaviour of single fibre since it is the basic constituent of the nonwoven. Hence, tensile tests of single fibre were performed to obtain the required material properties. Finally, a discontinuous parametric FE model was developed utilizing a newly developed subroutine, and material properties of the fabric constituents were calculated.

As nonwovens are anisotropic materials, the results obtained from simulations were presented for two principal directions of nonwoven materials: machine direction (MD) and cross direction (CD). These results were then compared with the experimental ones. 


\section{Material}

The material used in this study is $20 \mathrm{~g} / \mathrm{m}^{2}$ (or gsm) thermally-bonded nonwoven, composed of monocomponent polypropylene fibres (manufactured by FiberVisions, USA). Polypropylene is one of the most commonly used materials for nonwovens because of its chemical stability, good mechanical strength and low melting temperature [11]. The microstructure of the nonwoven fabric is presented in Fig. 1. Apparently, the fabric is composed of staple fibres having diameter of approximately $18 \mu \mathrm{m}$ with the length of $38.1 \mathrm{~mm}$ and linear density around 2.3 denier.

Fig. 1.

\section{Assessment of properties}

Properties of polymer fibres are characterized by the temperature variation during thermal bonding on nonwovens. Moreover, during manufacturing of nonwovens, stiffness of the material is increased by drawing fibres through the nozzle. Hence, it is not appropriate to use the properties of polypropylene available in literature for any sample size for modelling of these materials. Therefore, mechanical properties of fibres for FE modelling and orientation distribution of fibres were measured experimentally.

\subsection{Randomness of microstructure}

Low-density nonwovens (planer density less than $50 \mathrm{~g} / \mathrm{m}^{2}$ ) have a nonhomogeneous and discontinuous microstructure with voids and gaps. In order to develop a FE model for realistic simulation of these materials and their deformation and damage behaviours, it is essential to determine the randomness of their microstructure and implement it into the numerical model. This randomness was introduced into the model in terms of the orientation distribution function (ODF), which was obtained with an in-house software Nonwoven Anisotropy V1 (see [11] ). To do this, 20 $\mathrm{g} / \mathrm{m}^{2}$ nonwoven samples were prepared and their images were taken using scanning electron microscopy (SEM) and X-ray micro computed tomography (CT). A representative image, which illustrated the clearest view of fibres, was scanned with the algorithm based on the image-analysis techniques and computed the orientation distribution of fibres.

\subsection{Single-fibre properties}

Material properties of fabric were obtained based on the single-fibre behaviour as they are the basic constituent of the fabric. The parameters necessary for modelling the nonwovens are a flow curve (stress-strain behaviour in plastic region), the modulus of elasticity and the Poisson's ratio. These parameters were obtained by performing the tensile tests with single fibres at various loading rates (Fig. 2). The fibres were extracted from a free edge of the fabric; such extracted fibres 
present accurately material properties as the extraction process does not affect them. The edges of the fibres were attached to small pieces of cardboard for firm grip during testing and mounted on Instron ${ }^{\circledR}$ Micro Tester 5848 having Instron ${ }^{\circledR} \pm 5 \mathrm{~N}$ load cell. The fibre behaviour was determined for constant speed of cross-head movement (constant engineering strain rate).

Fig. 2 shows that the fibre behaviour observed at different strain rates is similar to that of typical polymer and is characterized by nonlinearity and high level of strain. A nonlinear deformation behaviour of a PP fibre consists of elastic and plastic stages. However, yield strain is approximately 0.1 , and fibre deformation is mainly governed by plasticity. Variation in position of stress-strain curves with a change in the strain rate is due to viscous effects in the material. In order to reduce the computation time, elasticity of the material could be assumed as linear instead of nonlinear for the numerical model, since the elastic range of deformation behaviour of a single fibre is small as compared to the plastic one.

\section{Fig. 2}

\subsection{Fabric properties}

The mechanical behaviour of the fabric was determined by performing tensile tests on 20 $\mathrm{g} / \mathrm{m}^{2}$ PP fabric in two principal directions, $\mathrm{MD}$ and $\mathrm{CD}$ (MD coincides with the direction of conveyer belt during manufacturing of nonwovens and $\mathrm{CD}$ is perpendicular to MD in fabric plane). The obtained results of fabric tensile tests in MD and CD show a direction-dependent nonlinear behaviour of the fabric for these two directions (see experimental part of Fig. 5). The FE model has identical bond point shape, size, pattern and number of fibres as the tested fabric (Table 1). Therefore, these tests were used to verify the results obtained with the FE model.

\section{Development of FE model}

A FE model of the low-density nonwoven material was developed within the MSC. Marc ${ }^{\circledR}$ software package using MSC Patran pre-processor. A subroutine was written in Patran Command Language (PCL) employing the parametric modelling technique, in which software reads the code and generates the model as mentioned in [7]. This reduces significantly the effort for reformulating the model to incorporate the actual orientation distribution of fibres. 
The FE model of the material developed in this study consists of bond points connected by the linking fibres. Information about the dimensions of model, bond point geometry, pattern and number of fibres is given in Table 1; the total number of fibres used in the model was 1038 .

Bond points were modelled with shell elements (element type 139 in MSC. Marc ${ }^{\circledR}, 2010$ ) with thickness identical to those in the fabric. On the other hand, fibres were modelled as truss elements (element type 9 in MSC. Marc ${ }^{\circledR}, 2010$ ) which carry only compression or tension and do not have bending stiffness. This reflects the character of loading of real-life fabrics that cannot bear in-plane compression. In order to avoid convergence problems, each truss elements representing a fibre is a single element having a common node with a shell element of a bond point. To calculate the number of fibres to be modelled, first the total length of fibres in the fabric sample with dimensions equal to those of the FE model was calculated with the following relation:

$$
L_{\text {Fibre_total }}=\frac{m_{\text {Fibre }}}{\rho A} \text {, }
$$

where $\rho$ is the fibre material density, $A$ is the fibre's cross-sectional area, $m_{\text {Fibre }}$ is the mass of fibres within the modelled part of fabric. $m_{\text {Fibre }}$ was calculated by multiplying the fabric density $\left(20 \mathrm{~g} / \mathrm{m}^{2}\right.$ in this study) with the surface area of the modelled fabric sample. Then, the number of fibres to be modelled was calculated by the following relation:

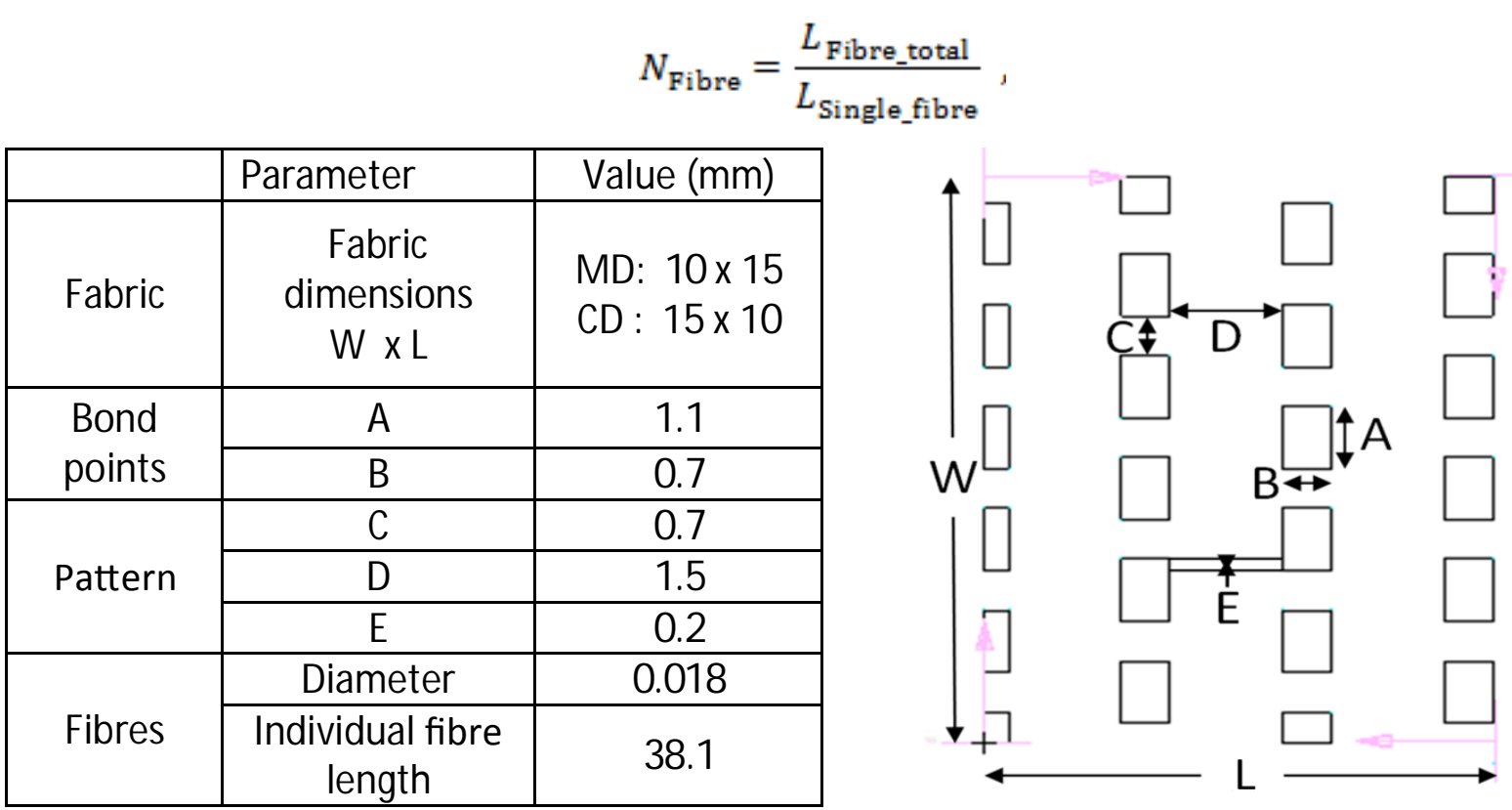

Table 1. Modelling parameters

where $N_{\text {Fibre }}$ is the number of fibres to be modelled and $L_{\text {single_fibre }}$ is the length of a staple fibre. As the fabric is made of staple fibres with the known length (see Table 1), the number of fibres to be modelled was determined by using above relations, and truss elements representing the fibres 
were introduced according to the ODF computed with Nonwoven Anisotropy V1. The crosssectional area, obtained from the known diameter of an individual fibre, is assigned to each truss element. All these calculations including the introduction of truss elements into the model were performed by the subroutine. In order to mimic the actual tensile test conditions of the fabric, the FE model was developed with the boundary conditions identical to those of the tensile tests. A constant-loading-speed-condition with magnitude equal to that of the actual tensile tests was applied in the direction of the tensile loading to the nodes on edge A-B of the model (Fig. 3) while all other degrees of freedom of these nodes were fixed. Nodes on edge $\mathrm{Y}-\mathrm{Z}$ of the model were fully constrained.

\section{Fig. 3.}

\section{Results and Discussion}

In order to evaluate efficiency of the developed model, tensile tests of $20 \mathrm{~g} / \mathrm{m}^{2}$ thermally bonded nonwoven (Fig. 1) were performed and used for comparison with simulation results. Those tests indicated that bond points changed their orientation at damage initiation but their deformation was negligible as compared to that of the fibrous matrix leading to its failure before the bond points. This phenomenon is due to a stiffer character of bond points. Besides, in the process of stretching, the fibres reorient towards the loading direction and increasing their participation in load bearing (Fig. 4a). Apparently, fibres fail when the applied stress reaches their strength threshold. As a result, due to a random character of microstructure and of the resulting load-transfer process, damage in the fabric is not continuous; it rather takes place gradually by means of failure of individual fibres. A similar pattern of damage initiation was observed in the FE model (Fig. 4b) with fibres exposed to higher stresses than the bond points. To compare the FE results with the obtained test data, the force-extension curves were derived based on computed stress and strain values (Fig. 5).

\section{Fig. 4.}

A difference between the results of tests and FE models can be attributed to the fact that inter-fibre friction and interaction is not introduced into the model; hence, the FE model gave a softer response than a real fabric. The surfaces of fibres are rough with some bumps and cracks, which increase friction between fibres. Moreover, during thermal bonding, some of the fibres close to bond points are exposed to relatively high temperature in addition to fibres located at bond points resulting in additional bonding with solidification as shown in Fig. 6. Such bonds between fibres in the fibrous 
matrix increase fabric stiffness, and would be introduced into the numerical model at the next stage

\section{Fig. 5.}

\section{Fig. 6.}

\section{Conclusion}

In this paper, damage initiation in low-density nonwovens is investigated using a developed numerical approach based on a micromechanical model. FE simulations were implemented employing a parametric modelling technique based on the anisotropic elastic-plastic material behaviour for two principle directions - MD and CD. Due to a complex microstructure, fibres were modelled directly according to the ODF, obtained from SEM images with the developed subroutine. Direct fibre modelling has an advantage of presenting a non-uniform microstructure with voids and gaps in it, making the model more accurate for simulating the behaviour of low-density nonwovens. It is shown that there is a good agreement between the developed FE model and experiments. Furthermore, damage initiation can be visualised with this model. Based on parametric studies, the effect of various manufacturing parameters, such as bond point shapes, patterns etc., on damage initiation in low-density thermally bonded nonwovens can be unveiled with the model introduced in this paper.

\section{Acknowledgement}

We greatly acknowledge the support by the Nonwovens Cooperative Research Centre of North Carolina State University, Raleigh, USA.

\section{References}

[1] W. Albrecht, H. Fuchs, W. Kittelmann, Nonwoven Fabrics: Raw Materials, Manufacture, Applications, Characteristics, Testing Processes, Wiley-VCH, Weinheim, 2003.

[2] S. Michielsen, B. Pourdeyhimi, P. Desai, Review of thermally point-bonded nonwovens: materials, processes, and properties, J. Appl. Polymer Sci., 99 (5) (2006) 489-2496. 
[3] S. Limem, S.B. Warner, Adhesive point-bonded spunbond fabrics, Textile Res. J. 75 (1) (2005) $63-72$.

[4] D.H. Mueller, M. Kochmann, Numerical modeling of thermobonded nonwovens, Int. Nonwovens J. 13 (1) (2004) 56-62.

[5] H.S. Kim, B. Pourdeyhimi, P. Desai, A.S. Abhiraman, Anisotropy in the mechanical properties of thermally spot-bonded nonwovens: experimental observations, Textile Res. J. 71 (11) (2001) 965-976.

[6] X. Hou, M. Acar, V.V. Silberschmidt, 2D finite element analysis of thermally bonded nonwoven materials: Continuous and discontinuous models, Comput. Mater. Sci. 46 (2009) 700-707.

[7] B. Sabuncuoglu, M. Acar, V.V. Silberschmidt, A parametric finite element analysis method for low-density thermally bonded nonwovens, Comput. Mater. Sci. In press (2012).

[8] E. Demirci, M. Acar, B. Pourdeyhimi, V.V. Silberschmidt, Dynamic response of thermally bonded bicomponent fibre nonwovens, App Mech. Mater. 70 (2011) 405-409.

[9] E. Demirci, M. Acar, B. Pourdeyhimi, V.V. Silberschmidt, Finite element modelling of thermally bonded bicomponent fibre nonwovens: Tensile behaviour, Comput. Mater. Sci. 50 (4) (2011) 1286-1291.

[10] E. Demirci, M. Acar, B. Pourdeyhimi, V.V. Silberschmidt, Anisotropic elastic-plastic mechanical properties of thermally bonded bicomponent fibre nonwovens, Conference Proceedings ASME 2010, Bienial Conference on Engineering Systems Design and Analysis (ESDA 2010), Turkey 12-17, 2010, Istanbul, Turkey. paper no. ESDA2010 - 24864

[11] E. Demirci, M. Acar, B. Pourdeyhimi, V.V. Silberschmidt, Computation of mechanical anisotropy in thermally bonded bicomponent fibre nonwovens, Comput. Mater. Sci., In press (2012). 


\section{Figure Captions}

Fig. 1. SEM image of thermally bonded nonwoven fabric $\left(20 \mathrm{~g} / \mathrm{m}^{2} \mathrm{PP}\right)$

Fig. 2. Mechanical behaviour of polypropylene fibre for three strain rates (diameter $18 \mu \mathrm{m}$ and gauge length $20 \mathrm{~mm}$ )

Fig. 3. Modelled Fabric

Fig. 4. Damage initiation in nonwovens: (a) microscopic image of nonwoven under tensile extension of $30 \%$ (Arrows indicate places of damage onset); (b) corresponding FE model

Fig. 5. Force-extension curves of FE model and tests for MD and CD

Fig. 6. Adhesion of fibres in fibrous matrix 


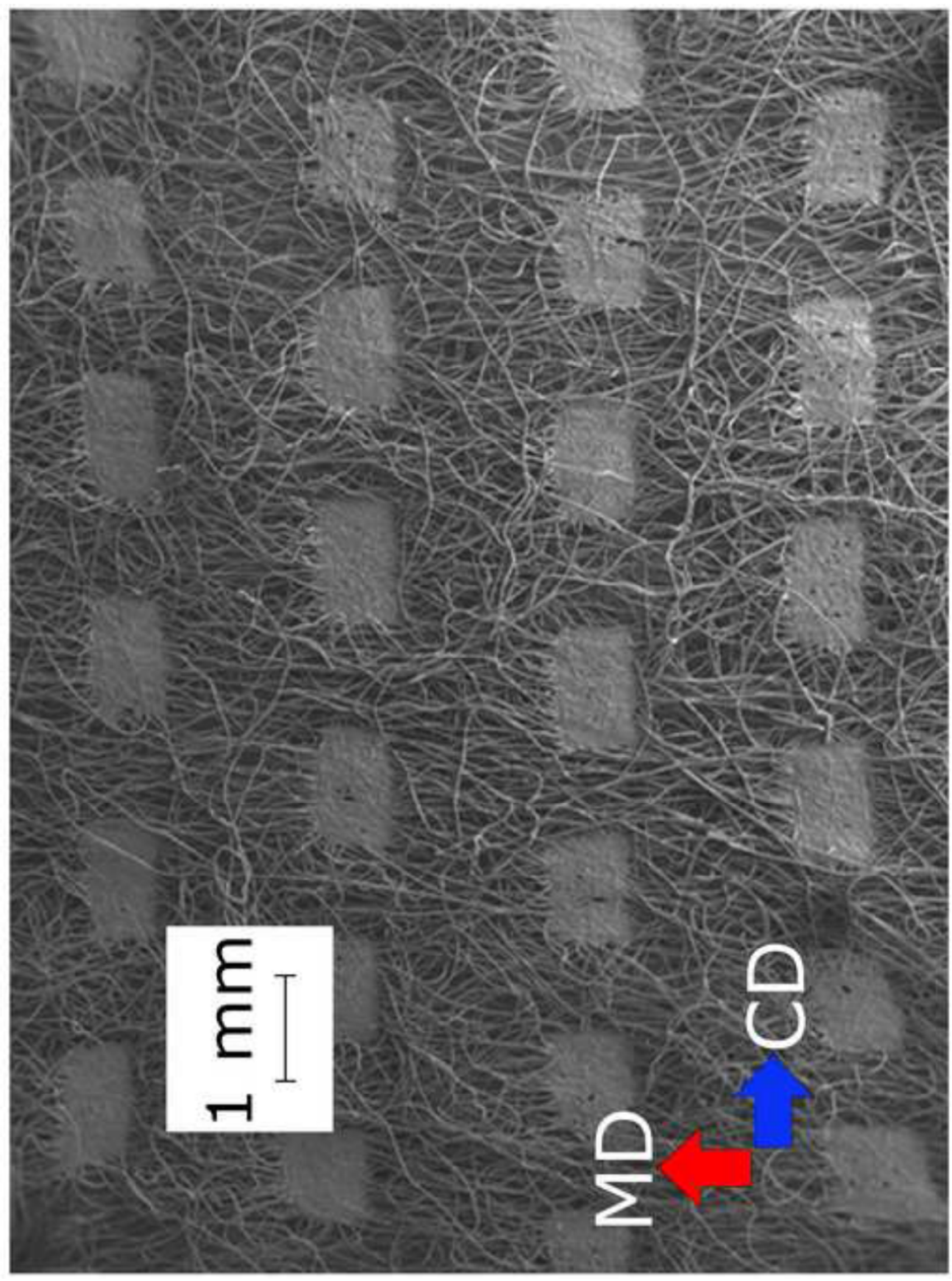




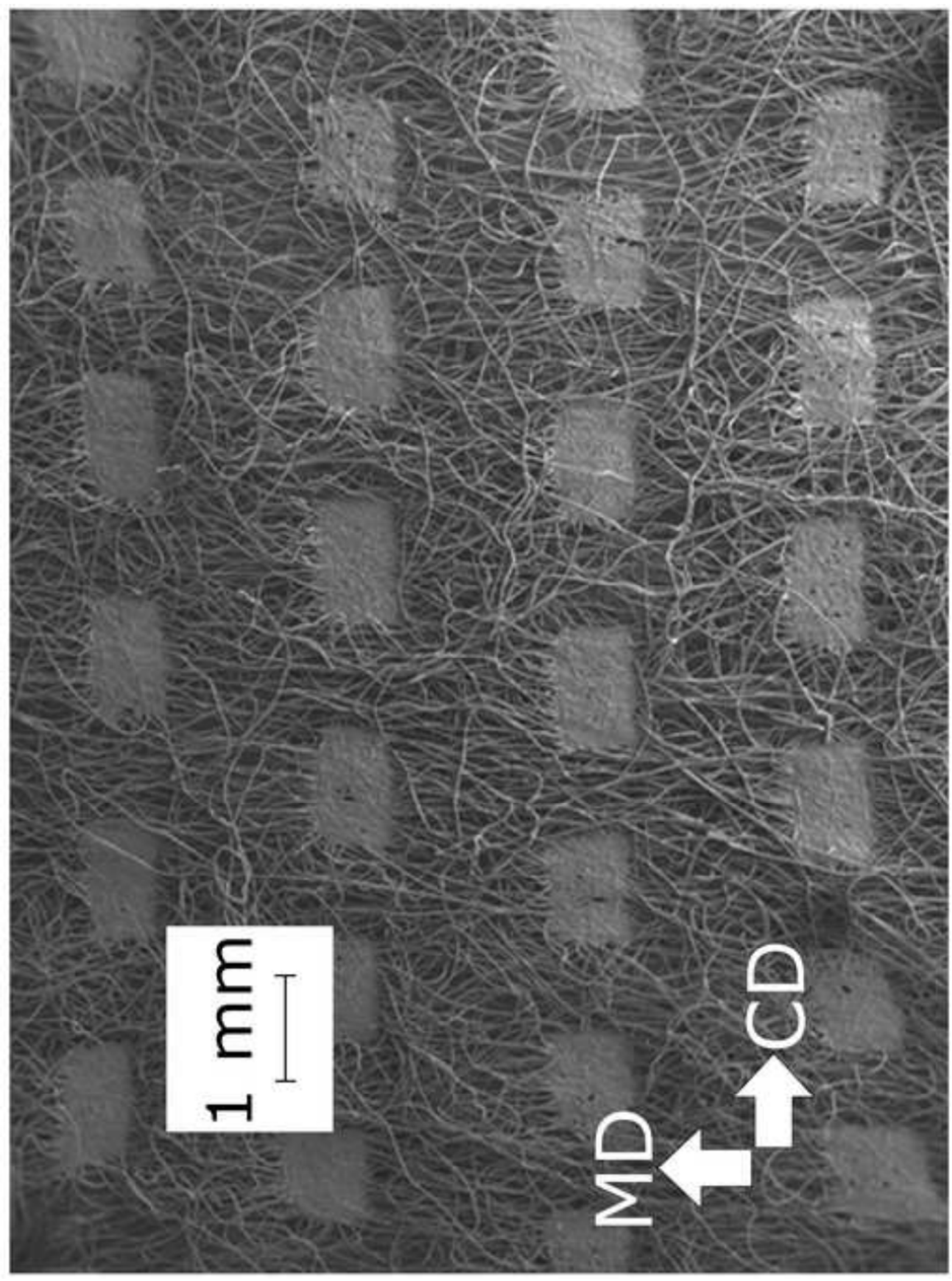




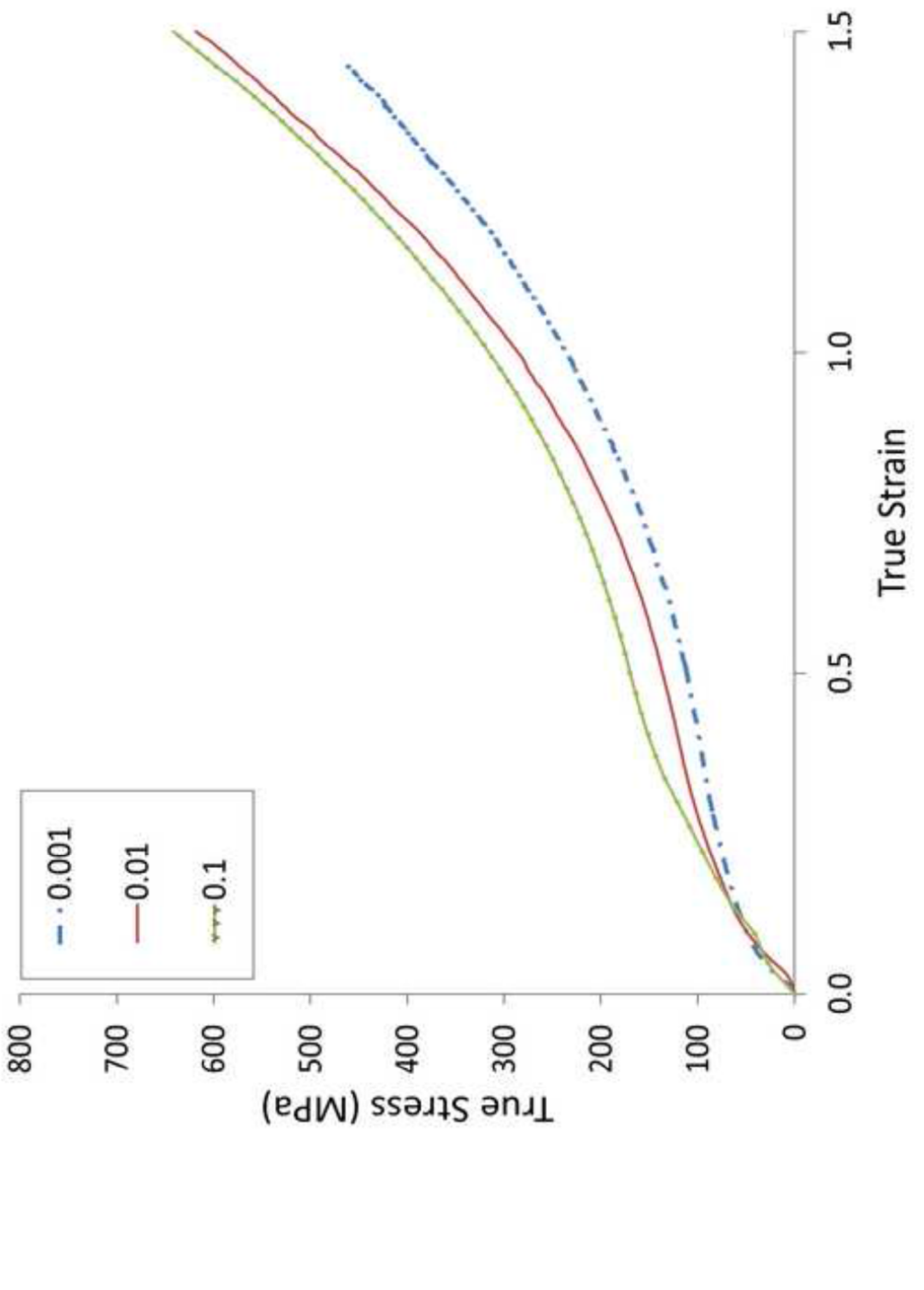




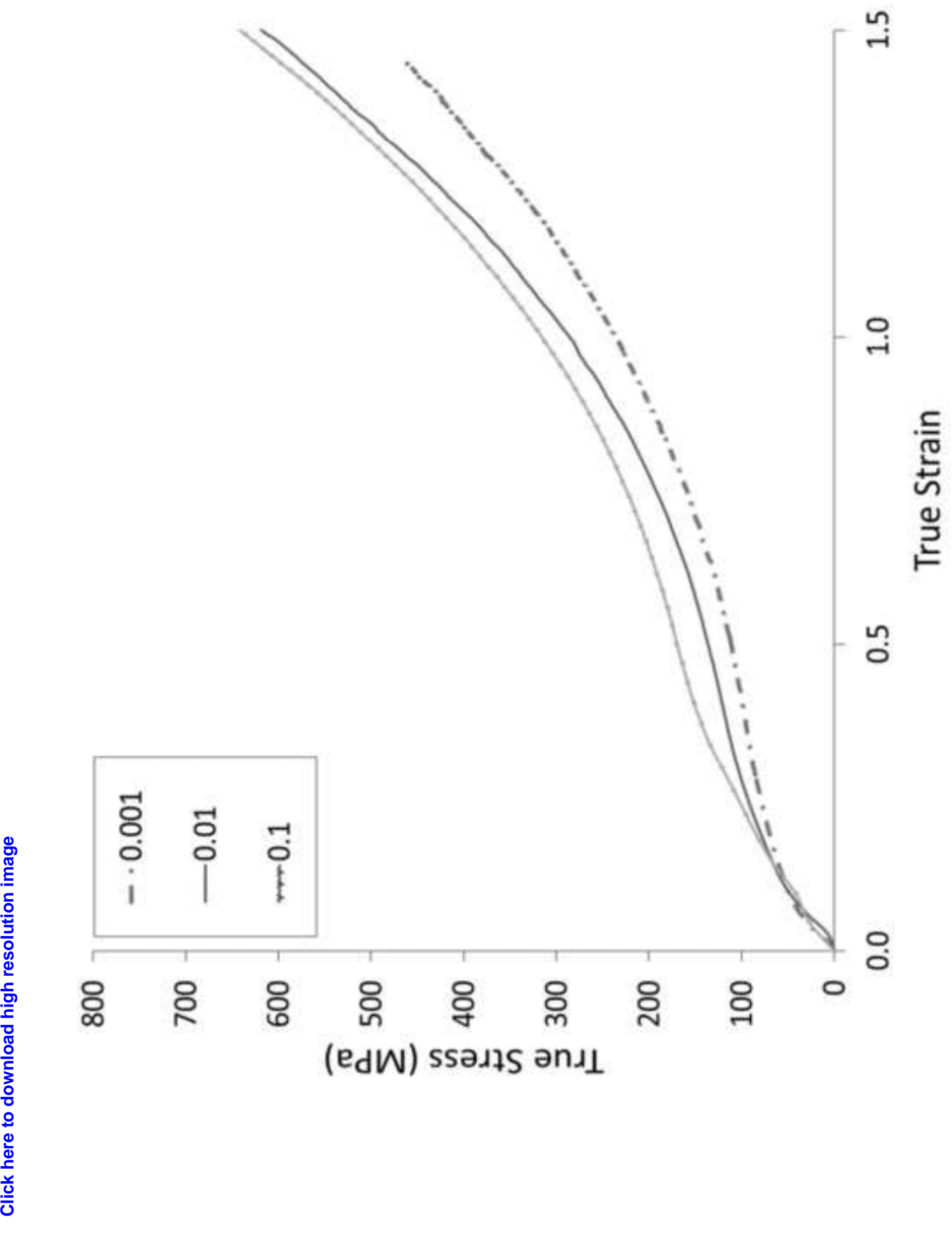




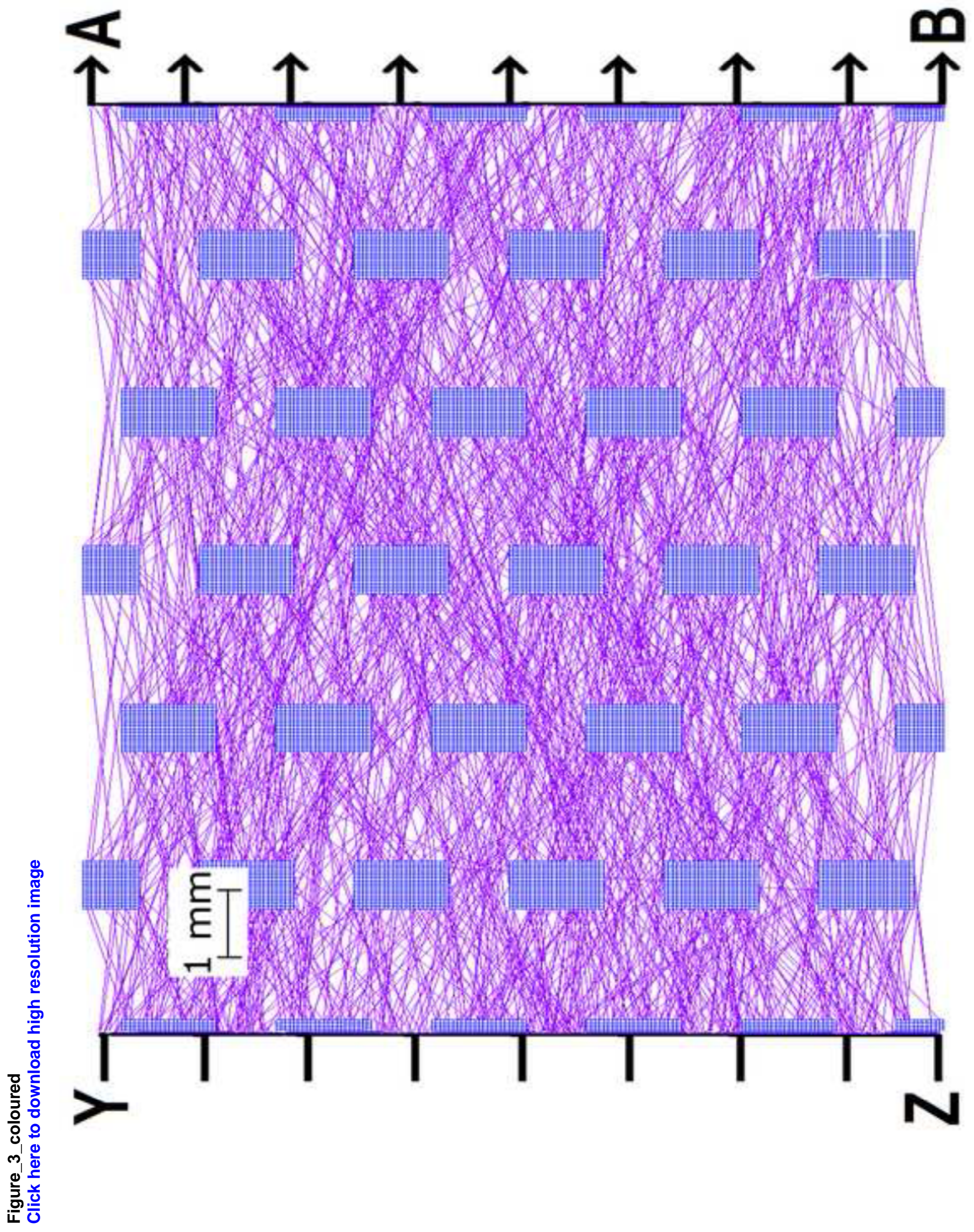




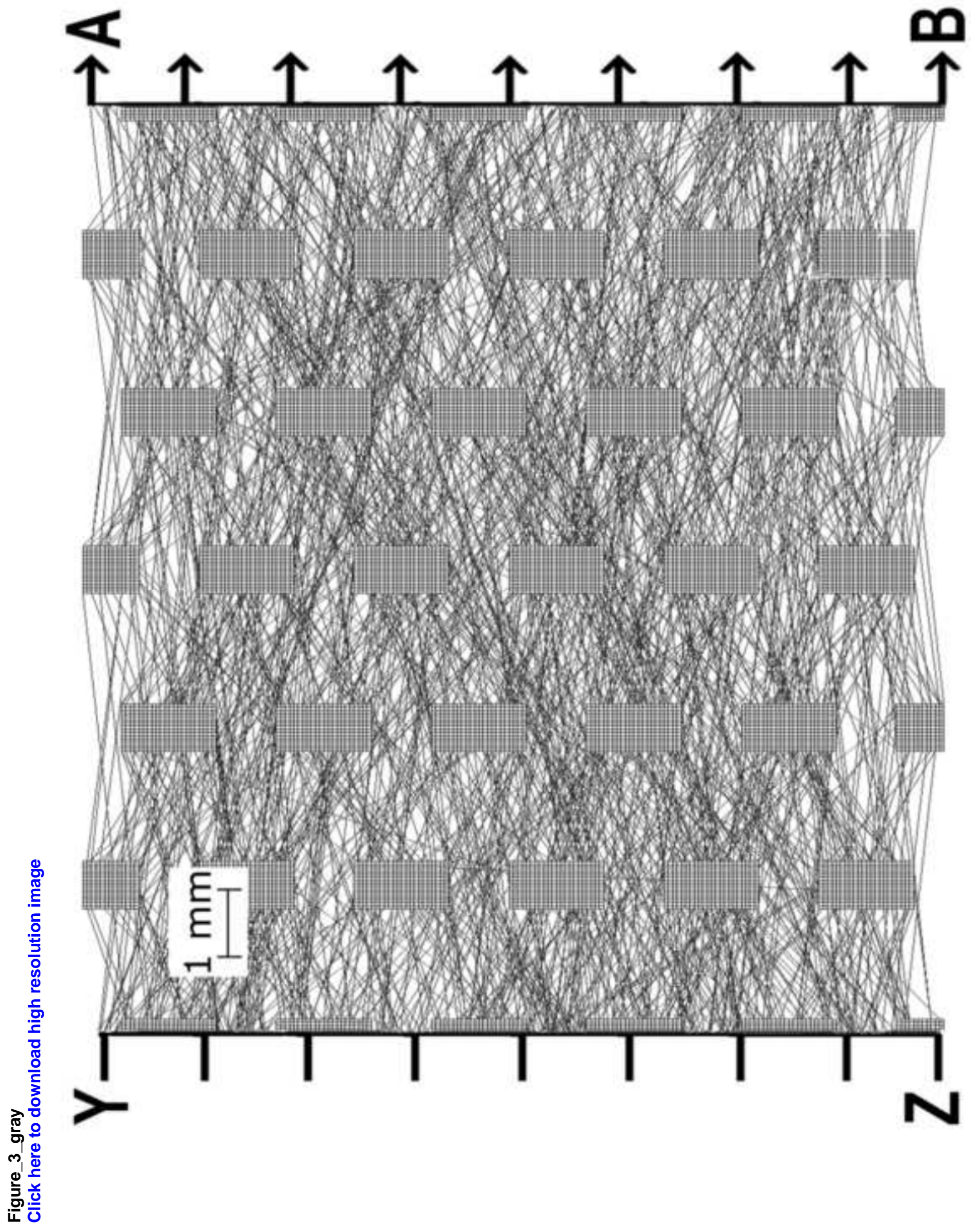




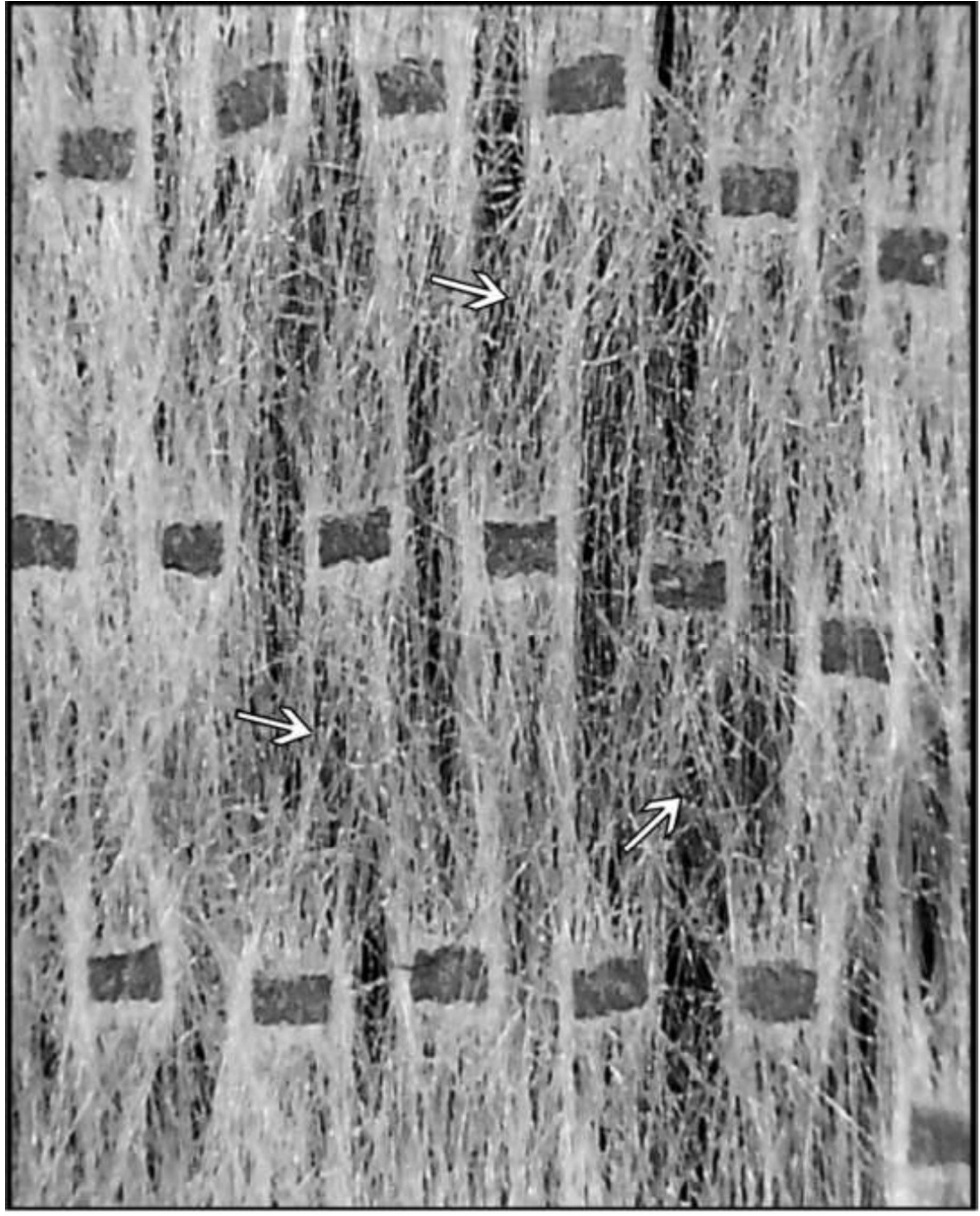




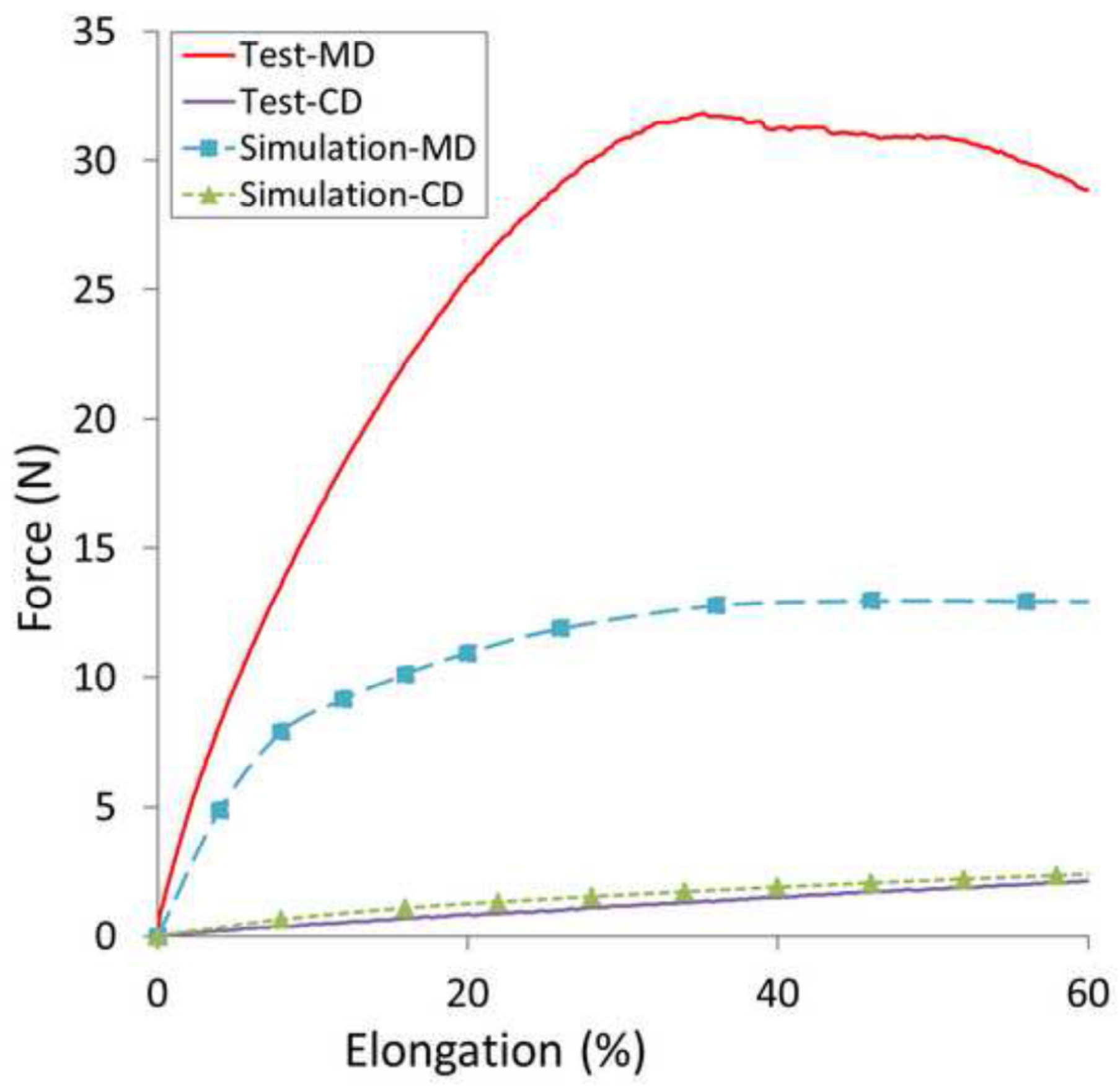




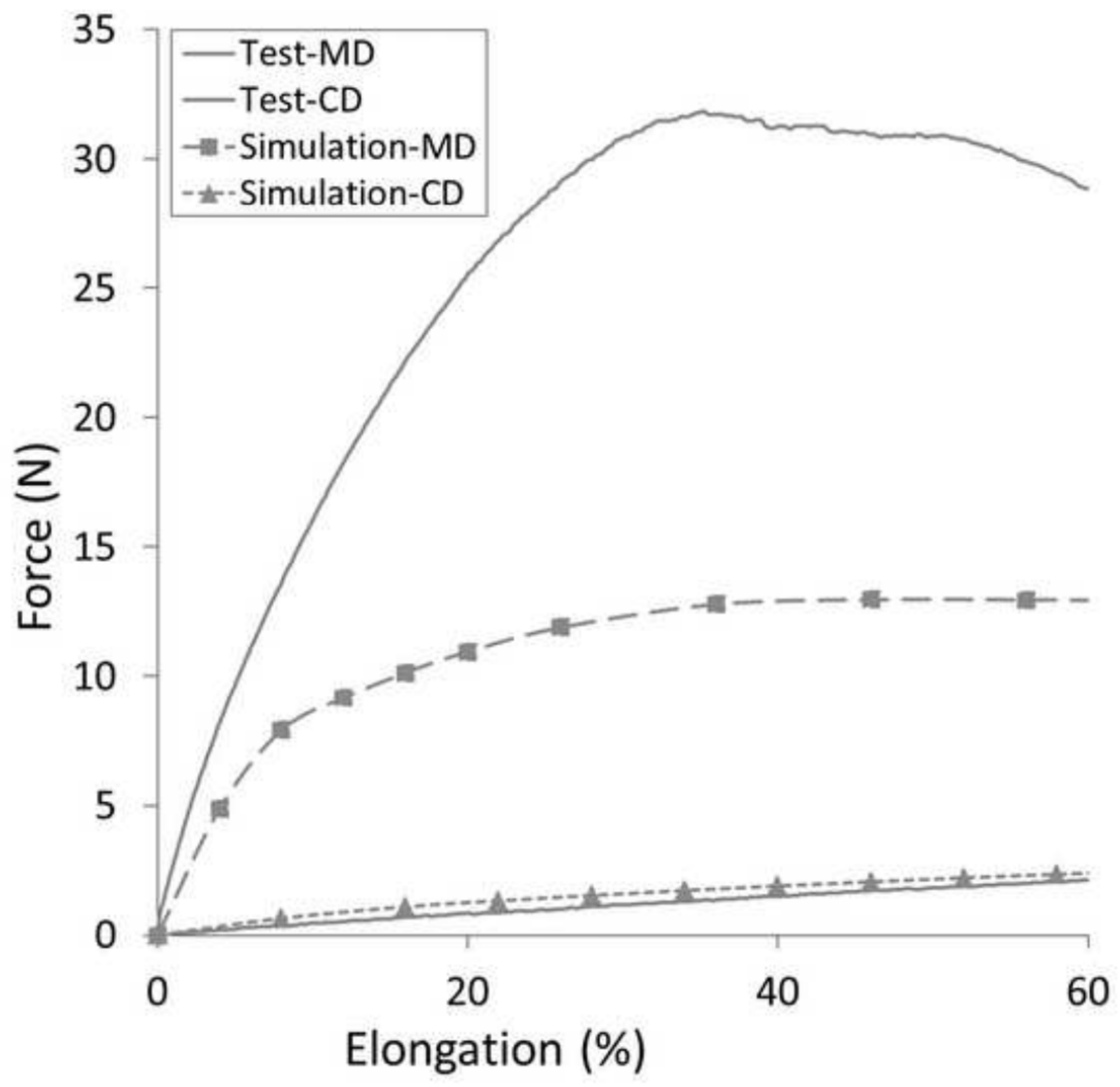




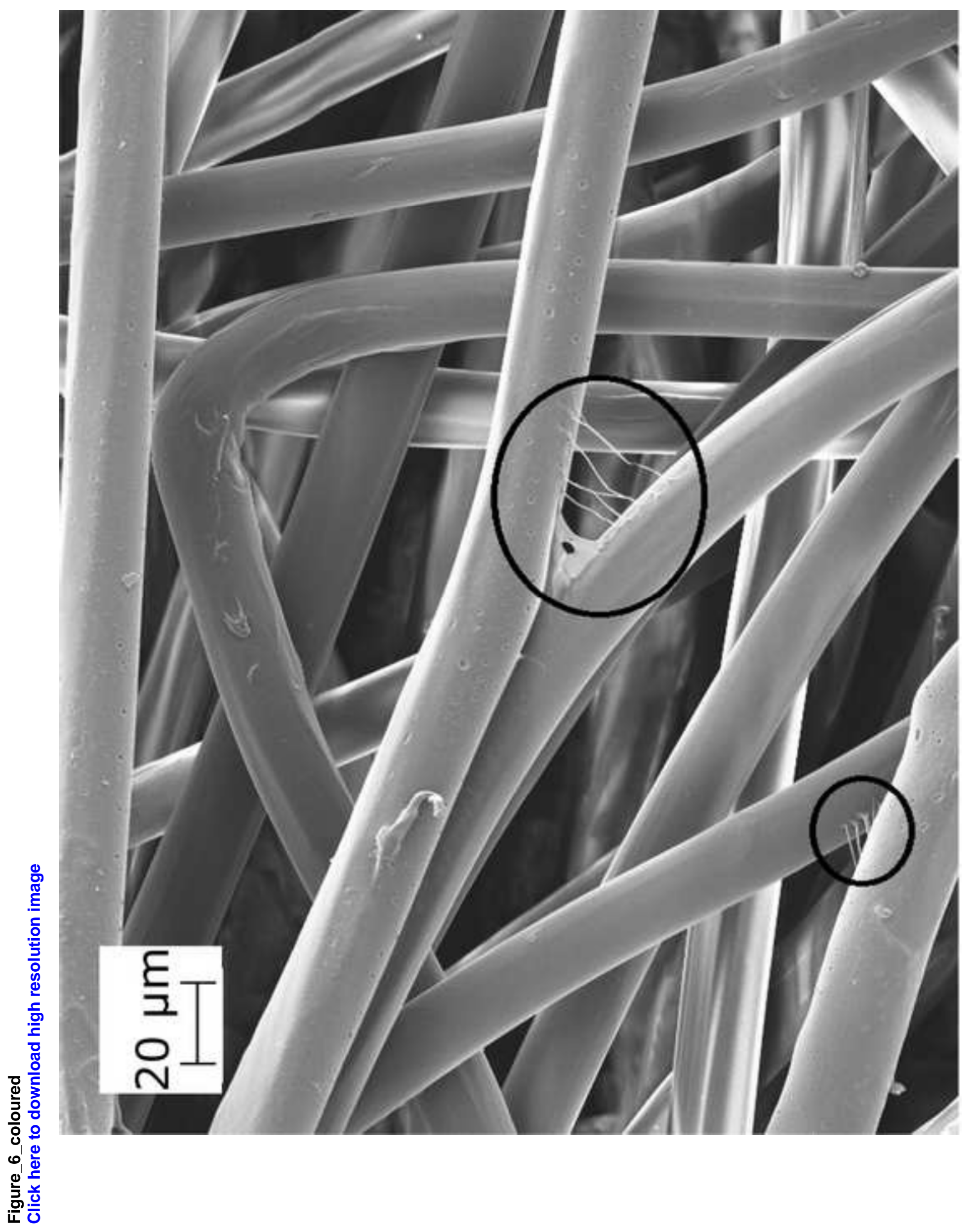




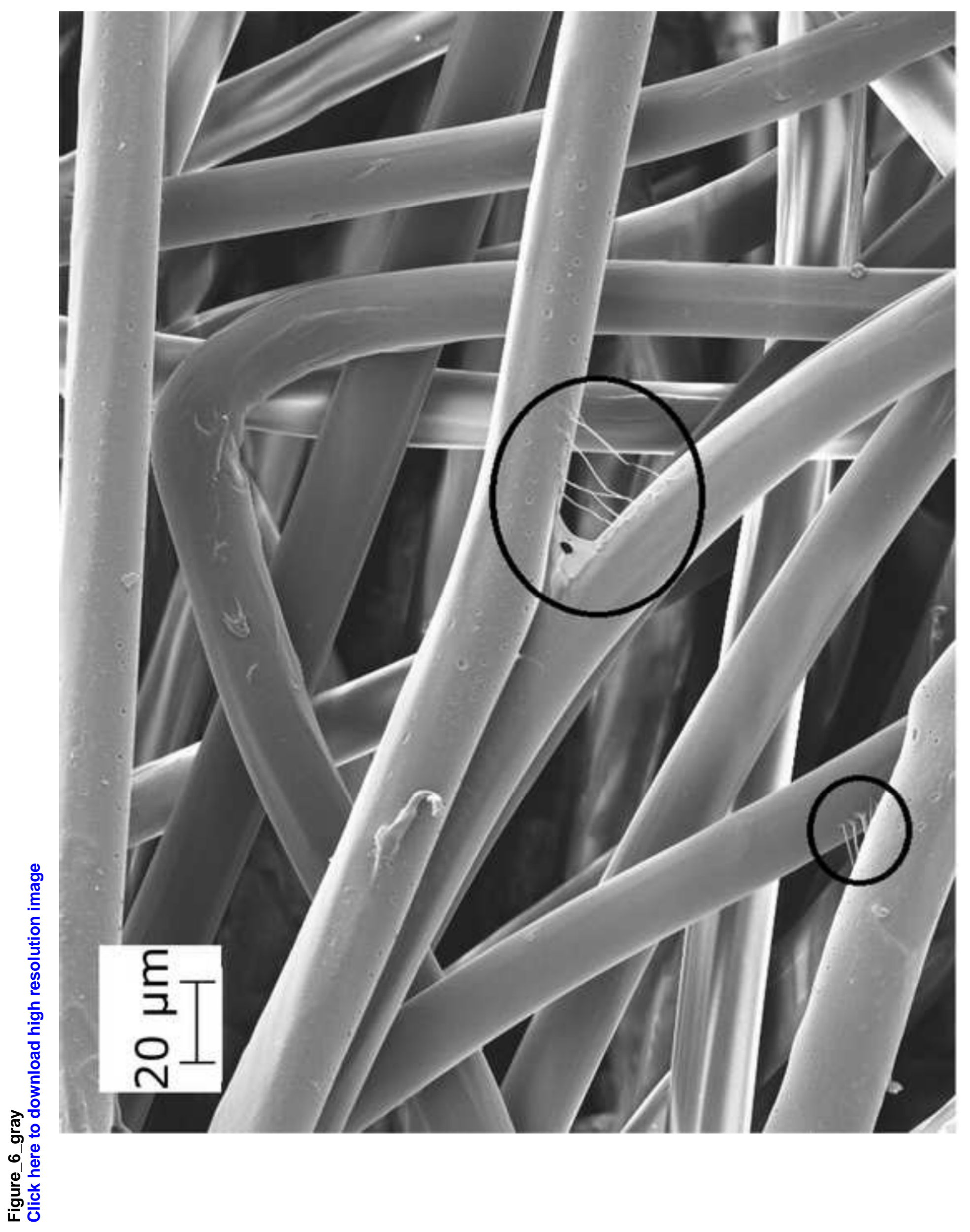

